\title{
Aberrant DNA methylation profiles of inherited and sporadic colorectal cancer
}

\author{
Nora Sahnane ${ }^{1}$, Francesca Magnoli ${ }^{1}$, Barbara Bernasconi ${ }^{1}$, Maria Grazia Tibiletti ${ }^{2}$, Chiara Romualdi ${ }^{3}$, \\ Monica Pedroni ${ }^{4}$, Maurizio Ponz de Leon ${ }^{4}$, Giulia Magnani ${ }^{4}$, Luca Reggiani-Bonetti ${ }^{5}$, Lucio Bertario ${ }^{6}$, \\ Stefano Signoroni ${ }^{6}$, Carlo Capella', Fausto Sessa ${ }^{1}$, Daniela Furlan ${ }^{1 *}$ and AIFEG
}

\begin{abstract}
Background: Aberrant DNA methylation has been widely investigated in sporadic colorectal carcinomas (CRCs), and extensive work has been performed to characterize different methylation profiles of CRC. Less information is available about the role of epigenetics in hereditary CRC and about the possible clinical use of epigenetic biomarkers in CRC, regardless of the etiopathogenesis. Long interspersed nucleotide element 1 (LINE-1) hypomethylation and gene-specific hypermethylation of 38 promoters were analyzed in multicenter series of 220 CRCs including 71 Lynch (Lynch colorectal cancer with microsatellite instability (LS-MSI)), 23 CRCs of patients under 40 years in which the main inherited CRC syndromes had been excluded (early-onset colorectal cancer with microsatellite stability (EO-MSS)), and 126 sporadic CRCS, comprising 28 cases with microsatellite instability (S-MSI) and 98 that were microsatellite stable (S-MSS). All tumor methylation patterns were integrated with clinicopathological and genetic characteristics, namely chromosomal instability (CIN), TP53 IosS, BRAF, and KRAS mutations.
\end{abstract}

Results: LS-MSI mainly showed absence of extensive DNA hypo- and hypermethylation. LINE-1 hypomethylation was observed in a subset of LS-MSI that were associated with the worse prognosis. Genetically, they commonly displayed G:A transition in the KRAS gene and absence of a CIN phenotype and of TP53 loss. S-MSI exhibited a specific epigenetic profile showing low rates of LINE-1 hypomethylation and extensive gene hypermethylation. S-MSI were mainly characterized by MLH1 methylation, BRAF mutation, and absence of a CIN phenotype and of TP53 loss. By contrast, S-MSS showed a high frequency of LINE-1 hypomethylation and of CIN, and they were associated with a worse prognosis. EO-MSS were a genetically and epigenetically heterogeneous group of CRCs. Like LS-MSI, some EO-MSS displayed low rates of DNA hypo- or hypermethylation and frequent G:A transitions in the KRAS gene, suggesting that a genetic syndrome might still be unrevealed in these patients. By contrast, some EO-MSS showed similar features to those observed in S-MSS, such as LINE-1 hypomethylation, CIN, and TP53 deletion. In all four classes, hypermethylation of ESR1, GATA5, and WT1 was very common.

Conclusions: Aberrant DNA methylation analysis allows the identification of different subsets of CRCs. This study confirms the potential utility of methylation tests for early detection of CRC and suggests that LINE-1 hypomethylation may be a useful prognostic marker in both sporadic and inherited CRCs.

Keywords: Colorectal cancer, LINE-1, Gene hypermethylation, Lynch syndrome, Microsatellite instability, Early onset colorectal cancer

\footnotetext{
*Correspondence: daniela.furlan@uninsubria.it

'Department of Surgical and Morphological Sciences, Section of Anatomic

Pathology, University of Insubria, Via O. Rossi, 9, 21100 Varese, Italy

Full list of author information is available at the end of the article
} 


\section{Background}

Aberrant DNA methylation, including both widespread demethylation as well as site-specific gene hypermethylation, deregulates the genome and contributes to the loss of tissue homeostasis observed in aging and in cancer. Changes in DNA methylation have been widely investigated in sporadic colorectal carcinoma (sCRC), and extensive work has been carried out both to characterize different methylation profiles of colorectal carcinoma (CRC) [1-4] and to investigate the possible clinical applications of epigenetic biomarkers for the early detection of $\mathrm{CRC}$, risk assessment, prognostication, and therapeutic opportunities [5-8].

Recently, whole-genome methylation analyses of CRCs, precursor lesions, and normal colorectal mucosa provided confirmation that aberrant DNA methylation is common in CRCs and occurs early in colorectal tumorigenesis. Cancer-specific de novo methylation has been detected in aberrant crypt foci $[9,10]$ as well as being extensively observed in the histologically normal colonic mucosa of patients predisposed to multiple serrated polyps, the proposed precursors of $\mathrm{CRC}$ with a $\mathrm{CpG}$ island methylator phenotype-high (CIMP-H) [11]. To date, it is widely accepted that CIMP-H is a distinct form of epigenomic instability in sporadic CRC $[2,12]$ which is strongly associated with a hypermutated profile [13], with $B R A F^{V 6 O 0 E}$ mutation [14] and with microsatellite instability (MSI) through epigenetic silencing of MLH1 $[15,16]$.

Genome-wide-DNA hypomethylation is the other early epigenetic alteration that has been observed in sporadic CRCs [17-19], and it has been associated with genomic and chromosomal instability (CIN) [20-23], as well as with the deregulation of gene transcription and activation of retrotransposons [24]. Recently, long interspersed nucleotide element 1 (LINE-1) hypomethylation has been recognized as an independent factor for increased cancer-related mortality and overall mortality in CRC patients [25-27]. In addition, some recent studies proposed this biomarker for familial cancer risk assessment, suggesting that LINE-1 hypomethylation is one of the distinguishing features of non-Lynch Syndrome familial CRC $[28,29]$ and that it is associated with early-onset CRC $[28,30]$.

However, until now, the role of epigenetics in hereditary and familial CRC has not been thoroughly explored, and its contribution toward carcinogenesis has not been characterized because accurate cancer genetics risk assessments are often lacking in the familial cases analyzed [30-32]. On the other hand, the evaluation of aberrant DNA methylation patterns in well-characterized inherited CRCs compared with those observed in sporadic CRCs could improve our knowledge of general mechanisms of epigenetics in colorectal carcinogenesis and help to identify common biomarkers for cancer risk assessment and for prognostication.

For this purpose, we determined both widespread hypomethylation as well as site-specific gene hypermethylation in a large and multicenter tumor series including 71 Lynch (Lynch syndrome (LS)) CRCs with an identified pathogenic germline mutation, 23 early onset CRCs (under 40 years) in which the main inherited CRC syndromes had been excluded, and 126 sporadic CRCs. All tumor methylation patterns were integrated with clinico-pathologic profiles and genetic characteristics, namely MSI and CIN status, TP53 loss and $B R A F$, and KRAS mutations.

\section{Results}

\section{Patient grouping, genetic, and clinico-pathologic evaluation}

Formalin-fixed and paraffin-embedded (FFPE) CRCs were collected from three Italian institutes and included (I) 71 CRCs showing MSI from Lynch patients (Lynch colorectal cancer with microsatellite instability (LSMSI); ORPHA 144) carriers of mismatch repair (MMR) germline mutations including 44 MLH1, 22 MSH2, 4 MSH6, and 1 EPCAM pathogenetic variants. In this subset of cases, only class 5 variants were considered as defined by International Society for Gastrointestinal Hereditary Tumors (InSiGHT) Variant Interpretation Committee (Mismatch Repair Gene Variant Classification Criteria, Version 1.9 August 2013); (II) 28 sporadic CRCs showing high microsatellite instability (S-MSI); (III) 98 sporadic CRCs without MSI (S-MSS). For sporadic cases, previously characterized for MSI, the presence of known hereditary cancer syndromes was excluded; (IV) 23 microsatellite stability (MSS) CRCs from patients younger than 40 years (early-onset colorectal cancer with microsatellite stability: EO-MSS) recruited through the specialized Colorectal Cancer Registry of Modena in the period 1984-2008. As recently reported by Magnani $G$ et al. [33], FAP (familial adenomatous polyposis; ORPHA 733), MAP (MYH associated polyposis; ORPHA 247798), and LS were excluded in these 23 cases, as well as specific clinicopathologic, genetic, and epigenetic features were examined. The clinico-pathological data are summarized in Table 1. Mean age of patients at diagnosis was 59.6 years. By definition, all EO-MSS were 40 years old or younger, whereas patients with both S-MSI and S-MSS were significantly older (mean value 70 years). The average age of CRC onset in LS patients was in the mid-late 40s (47.1 years), decades younger than that observed in the sporadic cohort. In all analyzed subsets, male patients were more numerous than females. According to the site, nearly all (93\%) S-MSI occurred proximal to the splenic flexure, as well as the majority (69 \%) of LS-MSI. 
Table 1 Main clinico-pathologic characteristics of CRCS

\begin{tabular}{|c|c|c|c|c|}
\hline & LS-MSI & S-MSI & S-MSS & EO-MSS \\
\hline & \multicolumn{4}{|c|}{ No. of tumors/total ${ }^{\mathrm{a}}(\%)$} \\
\hline \multicolumn{5}{|l|}{ Age } \\
\hline - Mean, years & 47.1 & 70.5 & 69.5 & 35.4 \\
\hline - Range, years & $30-78$ & $41-88$ & $41-91$ & $24-40$ \\
\hline \multicolumn{5}{|l|}{ Gender } \\
\hline - Female & $33 / 71(47)$ & $10 / 28(36)$ & 41/94 (44) & $7 / 23(30)$ \\
\hline - Male & 38/71 (53) & $18 / 28(64)$ & $53 / 94(56)$ & $16 / 23(70)$ \\
\hline \multicolumn{5}{|l|}{ Site } \\
\hline - Proximal colon & $36 / 52(69)$ & $26 / 28(93)$ & 27/98 (28) & $3 / 23(13)$ \\
\hline - Distal colon & $16 / 52(31)$ & $2 / 28(7)$ & $71 / 98(72)$ & $20 / 23(87)$ \\
\hline \multicolumn{5}{|l|}{ Histological type } \\
\hline - Mucinous & $21 / 67(31)$ & $18 / 28(64)$ & $7 / 98(7)$ & $3 / 23(13)$ \\
\hline - Medullary & $8 / 67(12)$ & 8/28 (29) & 0/98 (0) & $0 / 23(0)$ \\
\hline - Signet ring cell & 8/67 (12) & $4 / 28(15)$ & 4/98 (4) & $0 / 23(0)$ \\
\hline \multicolumn{5}{|l|}{ Tumor grade } \\
\hline - G1, G2 & $30 / 62(48)$ & $13 / 28(46)$ & $83 / 98(85)$ & $20 / 23(87)$ \\
\hline - G3 & $32 / 62(52)$ & $15 / 28(54)$ & 15/98 (15) & 3/23 (13) \\
\hline \multicolumn{5}{|l|}{ TNM stage } \\
\hline$-|-| \mid$ & $48 / 67(72)$ & $18 / 25(72)$ & $38 / 93(41)$ & $8 / 22(36)$ \\
\hline - III-IV & 19/67 (28) & $7 / 25(28)$ & 55/93 (59) & $14 / 22(64)$ \\
\hline \multicolumn{5}{|l|}{ Follow-up } \\
\hline - Alive & $39 / 53(74)$ & $22 / 26(85)$ & $50 / 94(53)$ & $15 / 23(68)$ \\
\hline - Died of disease & $14 / 53(26)$ & 4/26 (15) & 44/94 (47) & $7 / 23(32)$ \\
\hline
\end{tabular}

LS-MSI lynch syndrome CRC, S-MSI sporadic MSI CRC, S-MSS sporadic MSS CRC, EO-MSS early onset CRC

${ }^{a}$ Clinico-pathological characteristics are not available for all cases in each subset

On the contrary, S-MSS and EO-MSS mainly affected the distal colon (72 and $87 \%$ of the cases, respectively). According to the histopathological variants, mucinous adenocarcinomas defined as tumors with more than $50 \%$ of the lesion being composed of pools of extracellular mucin prevailed in S-MSI patients (18 out of 28 cases; $64 \%$ ) and were progressively less represented in LS-MSI, EO-MSS, and S-MSS $(31,13$, and $7 \%$ of the cases, respectively). A subset of unstable cases (15\% of S-MSI and $12 \%$ of LS, respectively) and 4 S-MSS (4\%) displayed signet ring cell differentiation, defined by the presence of more than $50 \%$ of neoplastic cells with prominent intracytoplasmatic mucin and nuclear displacement. Finally, as expected, medullary carcinoma characterized by sheets of neoplastic cells associated with prominent infiltration by intraepithelial lymphocytes, was observed only in the MSI cohort (29\% of S-MSI and $12 \%$ of LS-MSI, respectively). Lowintermediate and high-grade adenocarcinomas were equally represented both in LS-MSI and S-MSI, with a prevalence of "tumor," "nodes," "metastasis" (TNM) stages I and II (72 \%). Conversely, most EO-MSS (87 \%) and S-MSS (85\%) were low-intermediate grade neoplasms which were frequently diagnosed at advanced stages (64 and $59 \%$ of the cases, respectively).

\section{LINE-1 hypomethylation profiles}

LINE-1 methylation analysis was possible in 217 out of 220 CRCs and in all the 25 normal mucosa samples included in the study. In morphologically normal mucosa, the percentage of LINE-1 methylation was always higher than $62 \%$ (average $64.5 \pm 2 \%$ ) while in CRCs, it ranged from 24 to $68 \%$ (average $54.3 \pm 7.5 \%$ ). Distribution of LINE-1 methylation levels in the four subsets of tumors showed a significant decrease of LINE-1 methylation rate going from S-MSI to S-MSS CRC (average 59.4 \pm $5.6 \%$ versus $51.7 \pm 8 \%$, respectively, $p<0.001$ ), while intermediate methylation levels were observed in LSMSI and in EO-MSS CRC (average $56.1 \pm 5.6 \%$ and $54.2 \pm 7.6 \%$, respectively) (Fig. 1 ).

In order to consider LINE-1 methylation as a discrete variable, we applied the k-means algorithm using a supervised clustering analysis. This method clearly subdivided all tumors into four groups showing significant differences of LINE-1 methylation levels: L1 cluster (51 CRCs, mean $63.1 \%$ ), L2 cluster (63 CRCs, mean $57.2 \%$ ), L3 cluster (77 CRCs, mean $50.9 \%$ ), and L4 cluster (26 CRCs, mean $40.2 \%$ ) (Fig. 2a). Notably, L3 and L4 clusters showed a significantly higher percentage of S-MSS and EO-MSS than LS-MSI and S-MSI CRC (63 and $52 \%$ versus 35 and $21 \%$, respectively; $p=0.0002$ ) (Table 2).

Univariate survival analysis on the whole series stratified by the four LINE-1 groups demonstrated that LINE-1 hypomethylation was a strong negative prognostic factor, with L4 cluster patients showing a median survival of 35 months compared to 156, 102, and more than 300 months for L3, L2, and L1 clusters, respectively ( $p=0.0005$; Fig. $2 \mathrm{~b})$. Survival analysis focusing specifically on each tumor class confirmed that the L4 cluster was associated with a worse prognosis when considering MSS cancers only $(p=0.028)$, while a trend toward a significant statistical value was observed when considering LS-MSI CRC only $(p=0.09)$. The small number of cases belonging to S-MSI and EO-MSS did not allow a survival analysis within these groups.

We also examined correlations of LINE-1 methylation with all the clinico-pathological features of the tumors reported in Table 1, but no significant associations were found. Interestingly, multivariable analysis of survival using the Cox proportional hazards revealed that advanced TNM stage (III and IV) and absence of MSI and LINE-1 hypomethylation (L4 

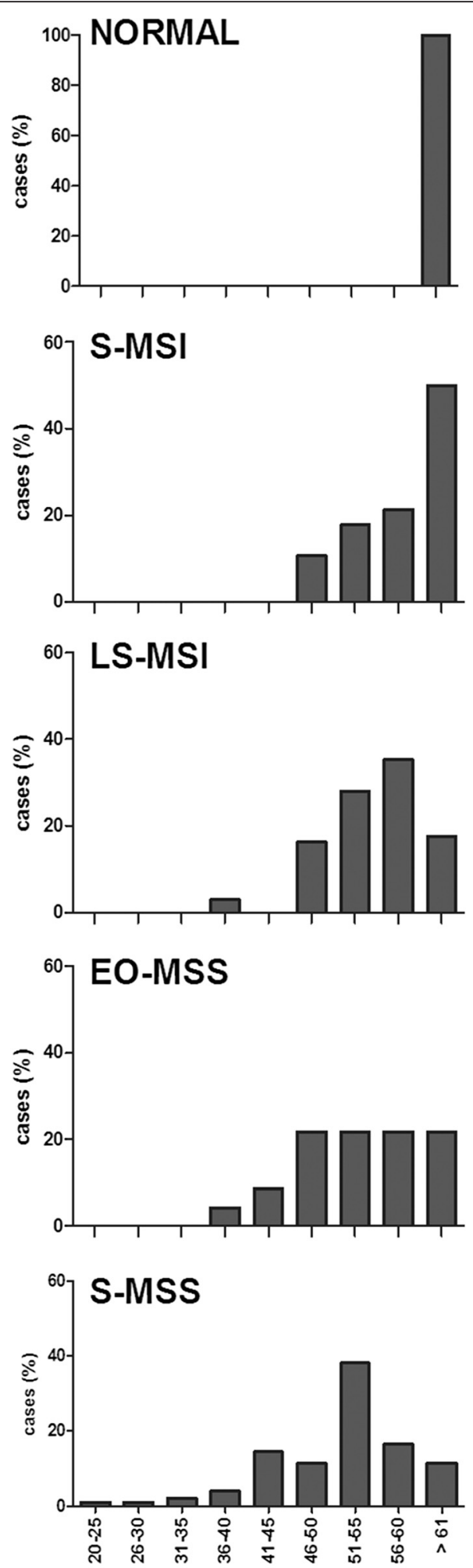

LINE-1 methylation
Fig. 1 LINE-1 methylation distribution in 25 samples of normal colonic mucosa and in 217 CRCs divided in four classes: S-MSI, LS-MSI, EO-MSS, and S-MSS. The distribution of LINE-1 methylation levels and the percentages of cases are shown on $x$-axis and $y$-axis, respectively

cluster) were independent factors of poor prognosis $(p=0.0018, p=0.0076$ and $p=0.0406$, respectively) (Table 3).

\section{Gene methylation profiles}

Thirty-eight promoter genes were examined by Methylation-Specific Multiplex Ligation-dependent Probe Amplification (MS-MLPA) in the 220 tumor samples.

LS-MSI and EO-MSS CRCs were characterized by significantly lower levels of gene-specific methylation compared with the remaining CRCs (average methylation percentage was $8.8 \%$ in both EO-MSS and LSMSI versus 15 and $29 \%$ in S-MSS and S-MSI, respectively; $p<0.0001)$. As evident from Fig. 3, gene methylation percentage was remarkably higher in $\mathrm{S}$ MSI compared with the other three CRC classes $(p<$ $0.0001)$ and it was positively correlated with MLH1 methylation $(p<0.0001)$ and with high levels of LINE-1 methylation (L1 and L2 clusters) $(p=0.006)$.

Unsupervised clustering of the promoter DNA methylation profiles identified three major clusters (Fig. 4) that were characterized by distinct methylation patterns. Cluster 1 (143 patients) displayed a very homogeneous profile showing significantly lower rates of methylation compared with cluster 2 (39 patients) and with cluster 3 (38 patients) $(p<0.0001)$. In particular, these two clusters exhibited an average of 9 and 11 hypermethylated genes (24 and $29 \%$ of gene methylation in cluster 2 and in cluster 3, respectively) and were considered as CIMP-high tumors, compared with non-CIMP cluster 1 showing an average of three hypermethylated genes (8\% of gene methylation). Cluster 1 included $83 \%$ of EO-MSS, $83 \%$ of LS-MSI, $60 \%$ of S-MSS, and only $21 \%$ of S-MSI. Cluster 1 CRCs were mainly characterized by hypermethylation restricted to only three genes that were extensively methylated in the whole series, namely GATA5, WT1, and ESR1. By contrast, cluster 2 and cluster 3 displayed extensive gene hypermethylation involving different genes. Cluster 2 was mainly composed of SMSS (Fig. 4) and showed a higher frequency of APC methylation than cluster $3(p=0.03)$.

The main features of cluster 3 were the considerably higher percentage of S-MSI $(68 \% ; p<0.0001)$ and a specific pattern of promoter methylation. In detail, six genes including MLH1, PAX6, PAX5, RARB, CDH13, and CHFR were significantly more often methylated in cluster 3 than in cluster $2(p<0.01)$. 


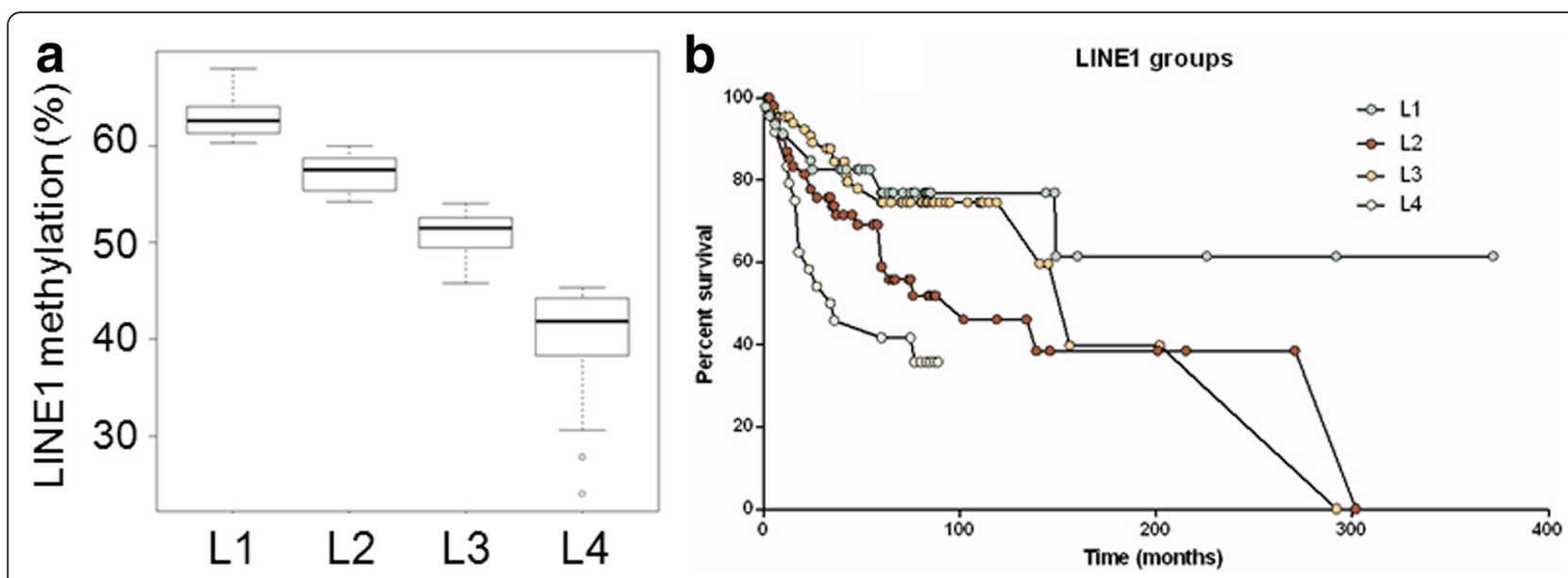

Fig. 2 a Supervised clustering analysis with k-means algorithm identifies four clusters showing significant differences of LINE-1 methylation levels: L1 (51 CRCs, mean $63.1 \%$ ), L2 cluster (63 CRCs, mean $57.2 \%$ ), L3 cluster (77 CRCs, mean $50.9 \%$ ), and L4 cluster (26 CRCs, mean $40.2 \%$ );

b Kaplan-Meier curves showing significantly different clinical outcome in patients stratified by tumor LINE-1 methylation groups $\left(p=5 \times 10^{-4}\right)$

\section{KRAS and BRAF mutation status}

KRAS and BRAF mutations were observed in 60/201 (29.8\%) and in 20/213 CRCs (9\%), respectively. As reported in Table 2, the only mutation observed in the BRAF gene was a V600E substitution that appeared to be closely related to S-MSI CRC (65\% of cases; $p<0.0001$ ) and with a high level of gene methylation $(p<0.0001)$. In contrast, BRAF mutations were rarely observed in S-MSS (2\%) and were never observed in LS-MSI and EO-MSS CRCs.

On the other hand, KRAS mutations were never detected in S-MSI, while they were identified in $43 \%$ of
LS-MSI, in $30 \%$ of S-MSS, and in $30 \%$ of EO-MSS CRC ( $p=0.0012$; Table 2 and Fig. 5a).

Interestingly, LS-MSI and EO-MSS CRCs showed almost exclusively $G$ to A transition because G12D or G13D were observed in 96 and in $84 \%$ of these tumors, respectively. KRAS G12V ( $\mathrm{G}$ to $\mathrm{T}$ transversion) was the only additional mutation in the remaining $4 \%$ of LS-MSI CRC and $16 \%$ of EO-MSS CRC. Conversely, the KRAS mutation spectrum observed in S-MSS CRC was significantly more heterogeneous as all the substitutions $\mathrm{G}>\mathrm{A}$, $\mathrm{G}>\mathrm{T}$, and $\mathrm{G}>\mathrm{C}$ were found with the following frequencies: $57,29$, and $14 \%$, respectively ( $p=0.001$; Fig. $5 \mathrm{~b})$.

Table 2 Summary of the genetic and epigenetic results in the four classes of CRC

\begin{tabular}{|c|c|c|c|c|c|}
\hline & LS-MSI & S-MSI & S-MSS & EO-MSS & $p$ value \\
\hline & \multicolumn{4}{|c|}{ No. of tumors/total ${ }^{a}(\%)$} & \\
\hline LINE-1 methylation & & & & & $2 \times 10^{-5}$ \\
\hline L1 (>60.1 \%) & $17 / 69(25)$ & $15 / 28(54)$ & 14/97 (14) & $5 / 23(22)$ & \\
\hline L2 (54.1-60 \%) & $28 / 69(40)$ & $7 / 28(25)$ & 22/97 (23) & $6 / 23(26)$ & \\
\hline L3 (45.8-54 \%) & $22 / 69(32)$ & $6 / 28(21)$ & $40 / 97(41)$ & 9/23 (39) & \\
\hline$\llcorner 4(<45.6 \%)$ & 2/69 (3) & $0 / 28(0)$ & $21 / 97(22)$ & $3 / 23(13)$ & \\
\hline Gene methylation & & & & & $10^{-12}$ \\
\hline Cluster 1 & $59 / 71(83)$ & $6 / 28(16)$ & $59 / 98(60)$ & 19/23 (83) & \\
\hline Cluster 2 & 7/71 (10) & $3 / 28(11)$ & 26/98 (27) & $3 / 23(13)$ & \\
\hline Cluster 3 & $5 / 71(7)$ & 19/28 (73) & 13/98 (13) & $1 / 23(4)$ & \\
\hline $\mathrm{CIN}$ & $0 / 9(0)$ & $1 / 6(17)$ & $12 / 16(75)$ & $3 / 6(50)$ & $4.9 \times 10^{-3}$ \\
\hline TP53 deletion & $0 / 9(0)$ & $1 / 6(17)$ & $6 / 16(31)$ & $3 / 6(50)$ & 0.10 \\
\hline KRAS mutation & $25 / 58(43)$ & $0 / 26(0)$ & 28/94 (30) & $7 / 23(30)$ & $1.2 \times 10^{-3}$ \\
\hline BRAF V600E mutation & 0/71 (0) & 18/27 (67) & 2/93 (2) & $0 / 23(0)$ & $<10^{-4}$ \\
\hline
\end{tabular}

LS-MSI lynch syndrome CRC, S-MSI sporadic MSI CRC, S-MSS sporadic MSS CRC, EO-MSS early onset CRC

${ }^{\text {a }}$ Data are not available for all cases in each subset 
Table 3 Multivariable survival analysis

\begin{tabular}{lccc}
\hline Variable & Hazard ratio & Cl 95 \% & $p$ value \\
\hline TNM stage (III-IV vs I-II) & 2.39 & $1.37-4.13$ & 0.0018 \\
MSS vs MSI status & 2.59 & $1.29-5.23$ & 0.0076 \\
LINE-1 hypomethylation (L4 vs L1) & 2.44 & $1.10-5.76$ & 0.0406 \\
\hline
\end{tabular}

\section{FISH results}

Additional file 1: Table S1 summarizes all the fluorescent in situ hybridization (FISH) results. Gains are more frequent than losses (42\% gain and $9.9 \%$ loss). In the majority of cases, gains of both regions on the same chromosome were detected suggesting the presence of polysomic clones in these cancers.

Monosomy, defined as the loss of both regions on the same chromosome, was observed in 10 cases. Only two regions were lost with respect to the reference probes: the TP53 locus in 10 cases (3 EO-MSS, 1 S-MSI, and 6 $\mathrm{S}-\mathrm{MSS}$; Table 2) and the $13 \mathrm{q} 14$ region in one case (SMSS \#59 in Additional file 1: Table S1). A CIN phenotype was observed in 16 out of 37 (43\%) CRCs. In detail, chromosomal instability was more frequently observed in S-MSS (12/16, $75 \%)$ and in EO-MSS (3/6 cases, $50 \%)(p=0.10)$. Conversely, only one out of six $(16.6 \%)$

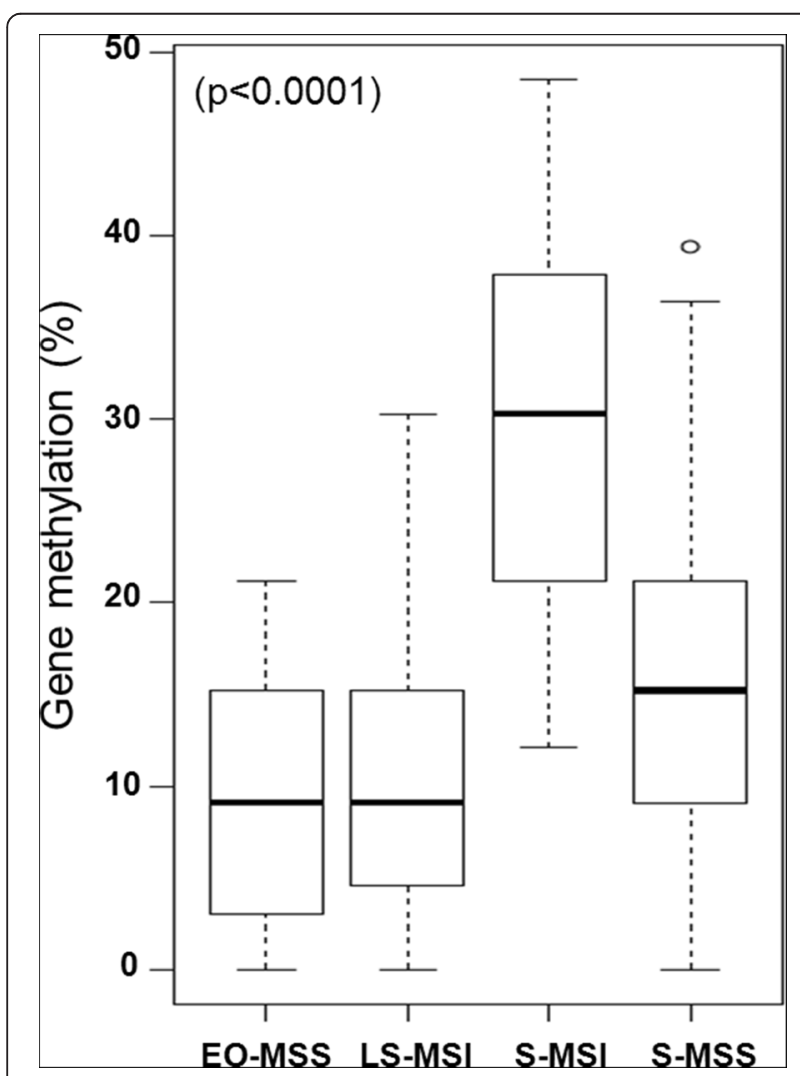

Fig. 3 Boxplots show a significantly higher level of gene-specific methylation in S-MSI compared with those observed in EO-MSS, LS-MSI, and S-MSS
S-MSI and none of nine LS-MSI showed the CIN phenotype (Table 2). Interestingly, low levels of LINE-1 methylation (L3 and L4 clusters) were mainly observed in CRCs showing TP53 loss $(p=0.03)$ and a CIN phenotype $(p=0.08)$ (Additional file 1: Table S1).

\section{Discussion}

In this research, global DNA hypomethylation as well as site-specific gene hypermethylation were examined in a multicenter series of sporadic and hereditary CRCs, using simple methods applicable to archival FFPE tissues, such as pyrosequencing and MS-MLPA. We chose to analyze a well-characterized series of inherited CRCs, including only LS patients carrying germline mutations in MMR genes. Likewise, we included in our study a selected group of patients with CRC, under the age of 40, in which the three main CRC-inherited syndromes (LS, MAP, and FAP) were excluded by genetic tests [33]. This is a crucial point since the available literature on aberrant DNA methylation in CRC is mostly focused on sporadic tumors while the role of epigenetics in hereditary and early onset CRCs is poorly known, also because specific cancer syndromes are not often accurately defined and there is no consensus about the age that defines young-onset CRC [30-32]. Actually, there is increasing attention for translation of epigenetic research into prevention and treatment of tumors [34, 35]. Aberrant methylation analyses of both sporadic and hereditary CRCs appear to be a promising strategy to better understand epigenetic mechanisms that may be generally involved in colorectal carcinogenesis regardless of the etiopathogenesis and the natural history of the tumor.

Our study revealed that the lowest levels of LINE-1 methylation (L3 and L4 in Table 2) were mainly observed in MSS tumors suggesting that this epigenetic mechanism might play a major role in CRCs without a MMR defect. Accordingly, FISH analysis demonstrated that LINE-1 hypomethylation was positively associated with TP53 deletion $(p=0.03)$ and that a trend toward significance was observed between a CIN phenotype and the lowest levels of LINE-1 methylation $(p=0.08)$. Altogether, these findings are clearly consistent with several data sustaining a causal link between DNA hypomethylation and chromosomal instability [20-23].

As reported in Table 3, LINE-1 hypomethylation was positively associated with a poor prognosis and was an independent prognostic factor in multivariate analysis, together with TNM stage and MSI status. To the best of our knowledge, despite LINE-1 methylation having been associated with a poor clinical outcome in a long list of human tumors (see review by Baba et al., [25]), it remains controversial as to whether the LINE-1 methylation level in CRC is associated with tumor stage, and its use for prognostic purposes is still far away to being 


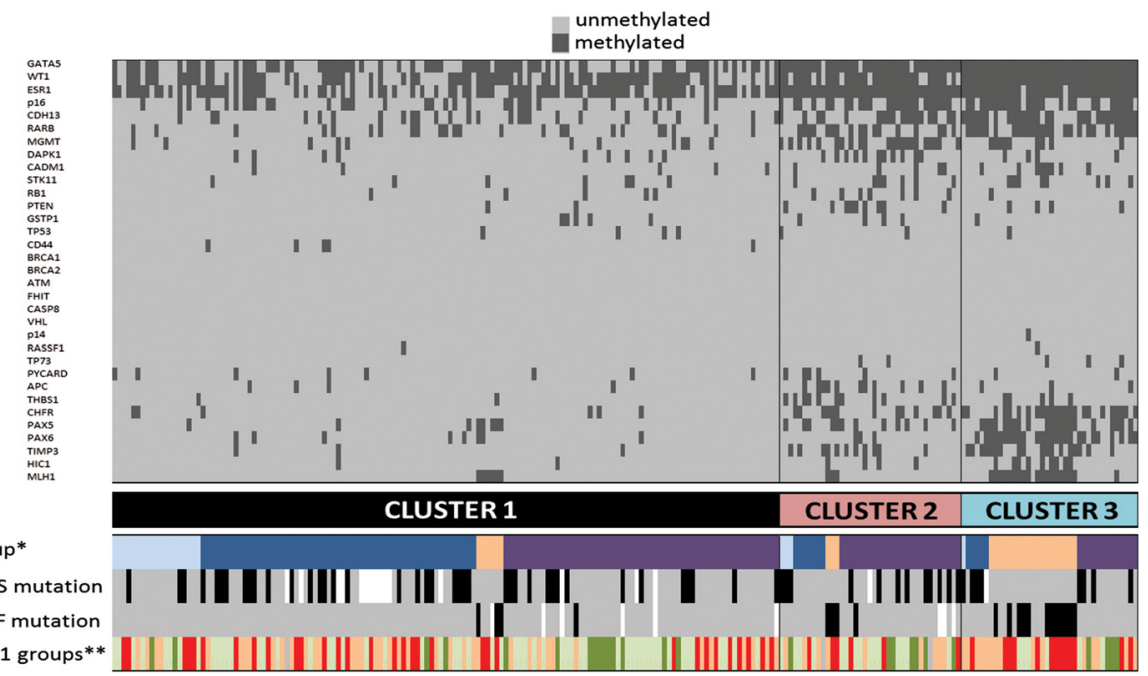

Fig. 4 Molecular subtypes of colorectal cancer. CRC are divided into three clusters by an unsupervised hierarchical algorithm. In the bottom panel, molecular data of tumor groups (*), namely LS-MSI (dark blue), EO-MSS (light blue), S-MSI (orange), and S-MSS (purple), are depicted. Black and gray cells indicate presence and absence of mutation, respectively; white cells represent data not evaluable. **: L1 (red), L2 (pink), L3 (light green), L4 (green)
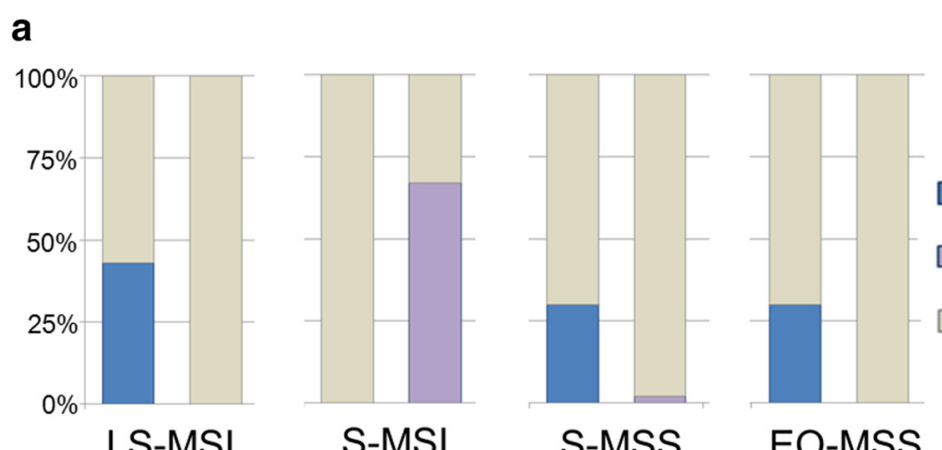

KRAS mutation

BRAF mutation

No mutation

b
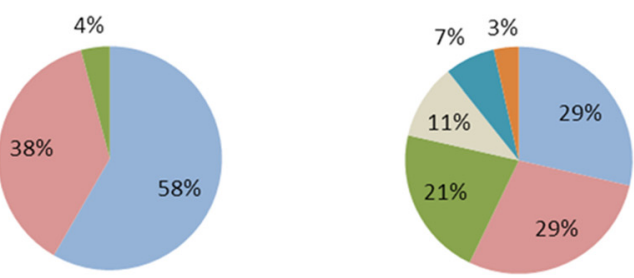

EO-MSS
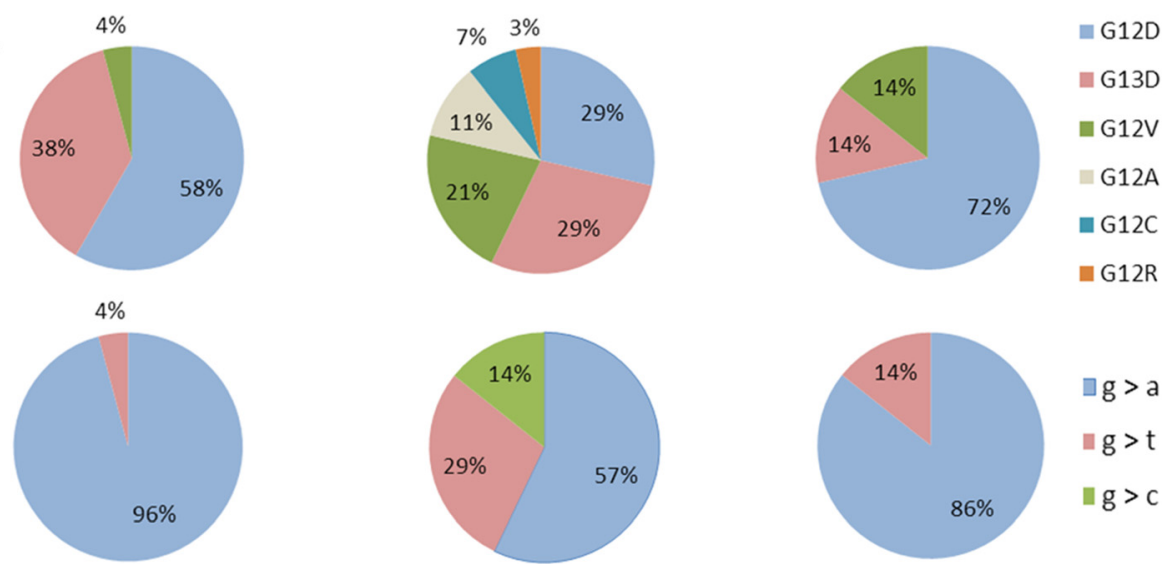

S-MSS

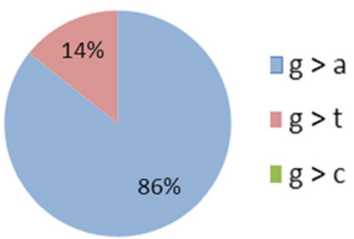

LS-MSI

EO-MSS

Fig. 5 Mutation analysis in the four tumor classes. a Frequencies of KRAS and BRAF mutation in CRCs. $\mathbf{b}$ Distribution of the amino acidic (upper) and the nucleotide substitutions (lower) in EO-MSS, LS-MSI, and S-MSS 
applied in clinical diagnostics. Sunami et al. [36] showed that LINE-1 demethylation is linearly correlated with TNM stage. However, in agreement with the results in our study, other recent works [27, 37] reported no relationships between the LINE-1 methylation levels and tumor stage, supporting the hypothesis that the LINE-1 hypomethylation may be initiated at an early stage of $\mathrm{CRC}$ remaining relatively stable throughout the longterm natural history of CRC development.

Interestingly, a subset of 24 out of 69 LS-MSI (Table 2) displayed L3 and L4 LINE-1 methylation levels and seemed to be more associated with a worse prognosis compared with the remaining LS-MSI $(p=0.08)$. Even if previous studies reported LINE-1 hypomethylation and MMR defects as two mutually exclusive markers in CRC [38], our data suggest that LINE-1 hypomethylation analysis might be useful to identify a subset of LS CRC showing a worse prognosis. In agreement with our findings, Inamura et al. [31] suggested that LINE-1 hypomethylation may be a valuable marker to find aggressive CRCs among generally indolent MSI CRCs. Our analysis extends and confirms these data in LS, suggesting a potential role of global DNA demethylation in a subset of MSI CRCs.

The role of DNA hypomethylation in EO-MSS was another point that we considered in light of recent studies reporting that a high degree of LINE-1 hypomethylation is a unique feature of young onset CRC without MSI [30]. Unlike data published by Antelo et al. [30], we did not find significantly lower levels of LINE1 methylation in EO-MSS compared to S-MSS. In our work, EO-MSS were mainly distal ( $87 \%$ of cases) and advanced CRCs (64\% of cases at stages III and IV) showing similar clinico-pathological profiles to those reported by Antelo et al. [30] and by other recent papers [39-41]. Interestingly, also the mean LINE-1 methylation levels in our series of EO-MSS were comparable to those reported by Antelo et al. [30] (54.2 \pm $7.6 \%$ versus $56.6 \pm 8.6 \%$ ). By contrast, the degree of LINE-1 methylation in older onset S-MSS was very different between the two studies, being significantly lower in our study $(51.7 \pm 8 \%$ versus $65.1 \pm 6.3 \%$ in the series published by Antelo et al. [30]). This observation suggests that the apparent discrepancy observed in our work is likely to be due to a different series of older onset S-MSS used in the comparative analysis and underlines the importance of the clinico-pathological and molecular features of the tumors selected for these evaluations. In conclusion, our results demonstrated comparable levels of LINE-1 methylation between EOMSS and S-MSS, suggesting that this marker alone is not a peculiar feature of young onset CRC without MSI and it is not enough to define a distinct clinical and molecular entity of CRCs.
With regard to hypermethylation at promoters of tumor suppressor genes, our study demonstrated that SMSI showed the highest percentages of hypermethylated genes $(p<0.0001)$, exhibiting frequent MLH1 methylation and a specific cluster of gene methylation with respect to the remaining tumors (cluster 3 in Fig. 4). This cluster was classified as CIMP-high and showed wellknown clinico-pathological and genetic features previously described for S-MSI with a CIMP phenotype [34]. Our analysis identified a second CIMP-high cluster (cluster 2 in Fig. 4) that was mainly composed of S-MSS. Cluster 2 was characterized by slightly lower levels of gene methylation compared with cluster 3 (on average 9 and 11 hypermethylated genes in cluster 2 and in cluster 3 , respectively) but no other clinico-pathologic or genetic features were specifically observed in these tumors. Our analysis does not allow to conclude whether cluster 2 may be considered a subset of CRCs with intermediate levels of gene methylation (CIMP-low) as recently reported by several studies and by genome scale DNA methylation profiling $[2,13,42]$. By contrast, clustering analysis in our study has clearly distinguished a third cluster (cluster 1 in Fig. 4) that was mainly composed of young onset CRCs, including both LS-MSI and EOMSS, that were characterized by the lowest rates of gene methylation compared with older onset CRC.

Altogether, these data are consistent with the hypotheses that aging is associated with an accumulation of aberrations in DNA hypermethylation in human tissues [43] and that S-MSI are a distinct form of epigenomic instability $[15,16]$ strongly associated with epigenetic silencing of $M L H 1$.

An interesting result of our work is that hypermethylation of three genes, namely ESR1, GATA5, and WT1, was very common in all four subsets of the CRCs examined. These findings are in agreement with previous reports sustaining that the hypermethylation of these genes is a cancer-specific event in gastrointestinal carcinogenesis since the early steps of neoplastic transformation [9, 44-47]. Recently, Valo et al. [48] emphasized the early appearance of epigenetic alterations in LS associated tumorigenesis, suggesting that methylation alterations may form carcinogenic fields in histologically normal mucosa of these patients. In this context, the opportunity to investigate the environmental influences on epigenetic changes represents a new challenge to understand the role of epigenetics on CRC pathogenesis. To date, several studies reported that lifestyle, aspirin use, microbiota, and inflammation likely influence colorectal tumorigenesis via altering the local tissue microenvironment, and epigenetics plays a key role in cellular response to microenviromental change [49-51]. In summary, available literature together with our current data strongly suggest the potential utility of gene hypermethylation tests for 
the early detection of $\mathrm{CRC}$, independently from the etiopathogenesis of the tumor.

The third issue addressed in our study was to correlate gene-specific methylation profiles with $B R A F$ or KRAS mutations (Table 2). In agreement with previous work, we found that $B R A F$ mutations were strongly associated with both $M L H 1$ methylations and with widespread gene hypermethylation confirming the well-established relationship of $B R A F$ mutations with MSI and with a CIMP$\mathrm{H}$ phenotype [14]. As expected, BRAF mutations were observed in $68 \%$ of S-MSI and in only $2 \%$ of S-MSS, whereas no BRAF mutations were observed in LS-MSI and in EO-MSS. This result clearly supports the use of this marker together with MLH1 methylation in order to discriminate LS-MSI from S-MSI [52] as well as confirming that the concurrence of $B R A F$ mutations with a CIMP-H phenotype is a specific feature of older onset CRC [15] but was absent in EO-MSS cancers.

KRAS mutations were found in three classes of CRC (LS-MSI, EO-MSS, and S-MSS) and were not associated with a specific methylation profile. Interestingly, both LSMSI and EO-MSS showed almost exclusively G:A transition, rather than $\mathrm{G}: \mathrm{T}$ or $\mathrm{G}: \mathrm{C}$ transversion mutations. In detail, G:A transition in cancer has been recognized as a DNA lesion caused by alkylating agents through the main mutagenic product such as $O^{6}$-methylguanine $\left(O^{6}\right.$-meG) [53]. The high frequency of G:A transitions in LS-MSI and in EO-MSS suggests that it is a common mutagenic mechanism in these tumors, differently from S-MSS where a more heterogeneous KRAS mutation spectrum was observed. This finding is novel and deserves to be developed at a genome-wide level, in light of recent knowledge about specific mutation signatures in cancer and related mutagenic mechanisms [54-56].

\section{Conclusions}

LS-MSI mainly show the absence of extensive DNA hypo- and hypermethylation, although LINE-1 hypomethylation may be observed in a subset of LS-MSI where they are associated with a worse prognosis. Genetically, they commonly display G: A transition in KRAS genes, an absence of a CIN phenotype and of TP53 loss.

Among sporadic CRCs, S-MSI exhibit a specific epigenetic profile showing low rates of LINE-1 hypomethylation and widespread gene hypermethylation. These tumors are mainly characterized by $M L H 1$ methylation, BRAF V600E mutation, and absence of CIN phenotype and of TP53 loss. By contrast, S-MSS show high frequency of LINE-1 hypomethylation and of CIN.

EO-MSS are a genetically and epigenetically heterogeneous group of CRC. Likewise LS-MSI, a subset of EO-MSS displays low rates of DNA hypo- or hypermethylation and a high frequency of G:A transition in the KRAS gene. On the contrary, some EO-MSS show similar features to those observed in S-MSS, such as LINE-1 hypomethylation, a CIN phenotype, and TP53 deletion. These results indicate that a subset of EOMSS resembles sporadic CRCs while the other subset displays some peculiar features of LS CRCs, suggesting that a genetic syndrome may not yet have been revealed in these patients.

Finally, our study confirms the potential utility of gene hypermethylation tests for the early detection of CRC and suggests that the LINE- 1 methylation assay may be a useful prognostic marker in both sporadic and hereditary CRCs.

\section{Methods}

Clinico-pathological study

Formalin-fixed and paraffin-embedded tissue samples from 220 surgically resected hereditary and sporadic CRCs were collected from three Italian institutes, namely the Department of Pathology of the Ospedale di Circolo-University of Insubria, Varese; the Unit of Hereditary Digestive Tract Tumors, Foundation IRCCS-INT, Milan; and the Department of Diagnostic Medicine, Clinical and Public Health, University of Modena and Reggio Emilia. All CRCs were histologically reviewed at the Department of Pathology of the Ospedale di CircoloUniversity of Insubria, according to the World Health Organization (WHO) classification of tumors of the digestive system [57] and the TNM staging system [58]. Outcome data were collected by consulting clinical records, the Tumor Registry of the Lombardy region (Italy), and the specialized Colorectal Cancer Registry of Modena and were available for 195 patients. This study was approved by the Ethics Committee of Ospedale di Circolo di Varese (n. 0037028) and was performed according to the Helsinki Declaration.

\section{LINE-1 methylation study}

The methylation status of LINE-1 was evaluated by bisulfite-PCR and pyrosequencing [59] in all the 220 CRCs and in twenty-five samples of histologically normal colonic mucosa. Genomic DNA was obtained from formalin-fixed and paraffin-embedded (FFPE) tissues using three representative 8-um-thick sections of each block. DNA was extracted after manual microdissection, using a QIAamp DNA FFPE tissue (Qiagen, Hilden, Germany). DNA bisulfite conversion was performed using Epitect kit (Qiagen, Hilden, Germany) according to the manufacturer's instructions. The LINE-1 pyrosequencing assay allowed the quantification of the mean methylation percentage of four consecutive $\mathrm{CpG}$ sites in the LINE-1 promoter region (GenBank accession number X58075), as previously reported [60]. Fully methylated and unmethylated DNA (Millipore, Billerica MA, 
USA) were used as positive and negative controls in each experiment.

\section{Gene methylation study}

Methylation analysis of a total of 38 gene promoters was performed in two replicates for each sample by MSMLPA using the SALSA MS-MLPA ME001-tumor suppressor-1, ME002-tumor suppressor-2, and ME011Mismatch Repair genes kit (MRC-Holland, Amsterdam, The Netherlands). MS-MLPA was performed according to the manufacturer's instructions, and data analysis was carried out with Coffalyser software v.8 (MRC-Holland). As previously described, we fully validated the sensitivity and specificity of these MS-MLPA assays; the presence or absence of promoter methylation were scored as discrete variables using the cutoff values formerly reported $[61,62]$.

\section{$B R A F$ and KRAS mutation analyses}

Mutations in codon 600 of the BRAF and codon 12 and 13 of the KRAS gene were analyzed in duplicate by PCRpyrosequencing using Anti EGFR MoAb response ${ }^{\circ}$ KRAS status kit and Anti EGFR MoAb response ${ }^{\bullet}$ BRAF status kit (Diatech Pharmacogenomics, Jesi, Italy) according to the manufacturer's instructions.

\section{FISH analysis}

In a subset of 37 CRCs including 9 LS-MSI, 6 S-MSI, 16 S-MSS, and 6 EO-MSS, interphasic FISH was performed on sections used for conventional histologic examination $(3-4 \mu \mathrm{m})$. The experiments were carried out as described elsewhere [63] using a panel of probes (Abbott, Chicago, USA) reported in Additional file 2: Table S2. For each FISH experiment, we used two probes mapping on to the same chromosome, one red labeled and one, the reference probe, green labeled. This FISH strategy permits the detection of loss and gain of specific regions. The cutoff values for losses and gains were defined using a panel of 10 paraffin-embedded control sections of non-neoplastic tissues and were calculated as $10 \%$ for gain and $12 \%$ for loss. Loss was considered when in each cell, red signals are less than green signals (reference probe); monosomy was defined when both analyzed regions on the same chromosome were loss. Gain was considered when more than two signals were observed in each cell. On the basis of these data, we defined a CIN phenotype when four or more gained regions (probes) were observed in the same colon cancer.

\section{Statistical analyses}

Association analyses were performed using the Fisher exact test, ANOVA, and the independent sample $t$ test. Supervised and unsupervised clustering analyses with k-means algorithm were used to analyze aberrant methylation data in order to distinguish different subsets of CRCs based on LINE-1 and gene methylation levels.

Patient survival was evaluated using the Kaplan-Meier method and statistically tested with the log-rank test. Patients who died within 1 month of surgery were excluded from the survival analyses. These analyses were performed with $\mathrm{R}$ software (https://www.r-project.org) with the mclust package [64, 65] and using GraphPad Prism V5.0 software. A $p$ value $\leq 0.05$ was considered statistically significant.

\section{Additional files}

Additional file 1: Table S1. Interphasic FISH results of 37 colorectal cancers. (XLS $40.5 \mathrm{~kb}$ )

Additional file 2: Table S2. Details of FISH probes used for CIN analysis. (DOCX $13.5 \mathrm{~kb}$ )

\section{Abbreviations}

ANOVA: analysis of variance; CIMP-H: CpG island methylator phenotype-high; CIN: chromosomal instability; CRC: colorectal carcinoma; EO-MSS: early-onset colorectal cancer with microsatellite stability; FAP: familial adenomatous polyposis; FISH: fluorescent in situ hybridization; FFPE: formalin fixed and paraffin embedded; InSiGHT: International Society for Gastrointestinal Hereditary Tumors; LINE-1: long interspersed nucleotide element 1; LS: Lynch syndrome; LS-MSI: Lynch colorectal cancer with microsatellite instability; MMR: mismatch repair; MSI: microsatellite instability; MS-MLPA: MethylationSpecific Multiplex Ligation-dependent Probe Amplification;

MSS: microsatellite stability; sCRC: sporadic colorectal carcinoma; S-MSI: sporadic colorectal cancer with microsatellite instability; S-MSS: sporadic colorectal cancer with microsatellite stability; TNM: "tumor," "nodes," "metastasis" (a cancer staging notation system); WHO: World Health Organization.

\section{Competing interests}

The authors declare that they have no competing interests.

\section{Authors' contributions}

NS, LB, MGT, MP, M PdeL, CC, and DF participated in the study design. FM, $C C, L R B$, and FS performed the clinico-pathological analysis. NS, BB, MP, GM, and SS performed the genetic and/or the molecular analysis. CR performed the statistical analysis. All authors contributed in the manuscript writing and approval of the final version.

\section{Authors' information}

AIFEG: info@aifeg.it. Italian Association for the Study of Familial and Hereditary Gastrointestinal Tumors-Collaborative study.

\section{Acknowledgements}

We thank the patients and clinicians contributing to this study. Giovanna Tagliabue is thanked for the collection of the follow-up of the patients. This study has been supported by the Epigenomics Flagship Project-EPIGEN (to D Furlan) (progetto finalizzato number 08934412) and by a grant from LILT (Lega Italiana per la lotta contro i Tumori).

\section{Author details}

${ }^{1}$ Department of Surgical and Morphological Sciences, Section of Anatomic Pathology, University of Insubria, Via O. Rossi, 9, 21100 Varese, Italy. ${ }^{2}$ Department of Pathology, Ospedale di Circolo, Varese, Italy. ${ }^{3} \mathrm{CRIB|}$ Biotechnology Center, University of Padova, Padua, Italy. ${ }^{4}$ Department of Diagnostic Medicine, Clinical and Public Health, University of Modena and Reggio Emilia, Modena, Italy. ${ }^{5}$ Department of Pathology, Policlinico di Modena, Modena, Italy. ${ }^{6}$ Unit of Hereditary Digestive Tract Tumours, Fondazione IRCCS - Istituto Nazionale dei Tumori Milan, Modena, Italy. 


\section{References}

1. Beggs AD, Jones A, El-Bahrawy M, Abulafi M, Hodgson SV, Tomlinson IP. Whole-genome methylation analysis of benign and malignant colorectal tumours. J Pathol. 2013;229:697-704.

2. Hinoue $T$, Weisenberger DJ, Lange $C P$, Shen $H$, Byun HM, Van Den Berg D, et al. Genome-scale analysis of aberrant DNA methylation in colorectal cancer. Genome Res. 2012;22:271-82.

3. Nejman D, Straussman R, Steinfeld I, Ruvolo M, Roberts D, Yakhini Z, et al. Molecular rules governing de novo methylation in cancer. Cancer Res. 2014;74:1475-83.

4. Yagi K, Akagi K, Hayashi H, Nagae G, Tsuji S, Isagawa T, et al. Three DNA methylation epigenotypes in human colorectal cancer. Clin Cancer Res. 2009;16:21-33.

5. Baylin SB, Jones PA. A decade of exploring the cancer epigenome-biological and translational implications. Nat Rev Cancer. 2011;11:726-34.

6. Lange CP, Campan M, Hinoue T, Schmitz RF, van der Meulen-de Jong AE, Slingerland $\mathrm{H}$, et al. Genome-scale discovery of DNA-methylation biomarkers for blood-based detection of colorectal cancer. PLoS One. 2012;7:e50266.

7. Lofton-Day C, Model F, Devos T, Tetzner R, Distler J, Schuster M, et al. DNA methylation biomarkers for blood-based colorectal cancer screening. Clin Chem. 2008;54:414-23.

8. Warren JD, Xiong W, Bunker AM, Vaughn CP, Furtado LV, Roberts $W L$, et al. Septin 9 methylated DNA is a sensitive and specific blood test for colorectal cancer. BMC Med. 2011:9:133.

9. Belshaw NJ, Pal N, Tapp HS, Dainty JR, Lewis MP, Williams MR, et al. Patterns of DNA methylation in individual colonic crypts reveal aging and cancer-related field defects in the morphologically normal mucosa. Carcinogenesis. 2010;31:1158-63.

10. Chan AO, Broaddus RR, Houlihan PS, Issa JP, Hamilton SR, Rashid A. CpG island methylation in aberrant crypt foci of the colorectum. Am J Pathol. 2002:160:1823-30.

11. Jass JR. Classification of colorectal cancer based on correlation of clinical, morphological and molecular features. Histopathol. 2007:50:113-30.

12. Toyota M, Ahuja N, Ohe-Toyota M, Herman JG, Baylin SB, Issa JP. CpG island methylator phenotype in colorectal cancer. Proc Natl Acad Sci U S A. 1999;96:8681-6.

13. Network CGA. Comprehensive molecular characterization of human colon and rectal cancer. Nature. 2012;487:330-7.

14. Fang M, Ou J, Hutchinson L, Green MR. The BRAF oncoprotein functions through the transcriptional repressor MAFG to mediate the CPG island methylator phenotype. Mol Cell. 2014;55:904-15.

15. Nazemalhosseini Mojarad E, Kuppen PJ, Aghdaei HA, Zali MR. The CpG island methylator phenotype (CIMP) in colorectal cancer. Gastroenterol Hepatol Bed Bench. 2013:6:120-8.

16. Sproul D, Meehan RR. Genomic insights into cancer-associated aberrant CpG island hypermethylation. Brief Funct Genomics. 2013;12:174-90.

17. Chalitchagorn K, Shuangshoti S, Hourpai N, Kongruttanachok N, Tangkijvanich $\mathrm{P}$, Thong-ngam D, et al. Distinctive pattern of LINE-1 methylation level in normal tissues and the association with carcinogenesis. Oncogene. 2004;23:8841-6.

18. Feinberg AP, Vogelstein B. Hypomethylation of ras oncogenes in primary human cancers. Biochem Biophys Res Commun. 1983;111:47-54.

19. Suter CM, Martin DI, Ward RL. Hypomethylation of $L 1$ retrotransposons in colorectal cancer and adjacent normal tissue. Int J Colorectal Dis. 2004;19:95-101.

20. Gasior SL, Wakeman TP, Xu B, Deininger PL. The human LINE-1 retrotransposon creates DNA double-strand breaks. J Mol Biol. 2006;357:1383-93.

21. Gaudet F, Hodgson JG, Eden A, Jackson-Grusby L, Dausman J, Gray JW, et al. Induction of tumors in mice by genomic hypomethylation. Science. 2003:300:489-92.

22. Rodriguez J, Frigola J, Vendrell E, Risques RA, Fraga MF, Morales C, et al. Chromosomal instability correlates with genome-wide DNA demethylation in human primary colorectal cancers. Cancer Res. 2006:66:8462-9468.

23. Yamada $Y$, Jackson-Grusby L, Linhart $H$, Meissner $A$, Eden $A$, Lin $H$, et al. Opposing effects of DNA hypomethylation on intestinal and liver carcinogenesis. Proc Natl Acad Sci U S A. 2005;102:13580-5.

24. Hur K, Cejas P, Feliu J, Moreno-Rubio J, Burgos E, Boland CR, et al. Hypomethylation of long interspersed nuclear element-1 (LINE-1) leads to activation of proto-oncogenes in human colorectal cancer metastasis. Gut. 2014;63:635-46.

25. Baba Y, Murata A, Watanabe M, Baba H. Clinical implications of the LINE-1 methylation levels in patients with gastrointestinal cancer. Surg Today. 2014:44:1807-16.

26. Miousse IR, Chalbot MC, Aykin-Burns N, Wang X, Basnakian A, Kavouras IG, et al. Epigenetic alterations induced by ambient particulate matter in mouse macrophages. Environ Mol Mutagen. 2014;55:428-35.

27. Murata A, Baba Y, Watanabe M, Shigaki H, Miyake K, Ishimoto T, et al. Methylation levels of LINE-1 in primary lesion and matched metastatic lesions of colorectal cancer. Br J Cancer. 2013;109:408-15.

28. Baba Y, Huttenhower C, Nosho K, Tanaka N, Shima K, Hazra A, et al. Epigenomic diversity of colorectal cancer indicated by LINE-1 methylation in a database of 869 tumors. Mol Cancer. 2010;9:125.

29. Goel A, Xicola RM, Nguyen TP, Doyle BJ, Sohn VR, Bandipalliam P, et al. Aberrant DNA methylation in hereditary nonpolyposis colorectal cancer without mismatch repair deficiency. Gastroenterol. 2010;138:1854-62.

30. Antelo M, Balaguer F, Shia J, Shen Y, Hur K, Moreira L, et al. A high degree of LINE-1 hypomethylation is a unique feature of early-onset colorectal cancer. PLoS One. 2012;7:e45357.

31. Inamura K, Yamauchi M, Nishihara R, Lochhead P, Oian ZR, Kuchiba A, et al. Tumor LINE-1 methylation level and microsatellite instability in relation to colorectal cancer prognosis. J Natl Cancer Inst. 2014;106:9.

32. Kirzin S, Marisa L, Guimbaud R, De Reynies A, Legrain M, Laurent-Puig $P$, et al. Sporadic early-onset colorectal cancer is a specific sub-type of cancer: a morphological, molecular and genetics study. PLoS One. 2014:9:e103159.

33. Magnani G, Furlan D, Sahnane, N., Reggiani Bonetti L, Domati F, and Pedroni M. Molecular features and methylation status in early onset (=40 year) colorectal cancer: a population based, case-control study. Gastroenterology Research and Practice 2015; in press.

34. Bardhan K, Liu K. Epigenetics and colorectal cancer pathogenesis. Cancers (Basel). 2013;5:676-713

35. Colussi D, Brandi G, Bazzoli F, Ricciardiello L. Molecular pathways involved in colorectal cancer: implications for disease behavior and prevention. Int J Mol Sci. 2013;14:16365-85.

36. Sunami E, de Maat M, Vu A, Turner RR, Hoon DS. LINE-1 hypomethylation during primary colon cancer progression. PLoS One. 2011;6:e18884.

37. Matsunoki A, Kawakami K, Kotake M, Kaneko M, Kitamura H, Ooi A, et al. LINE-1 methylation shows little intra-patient heterogeneity in primary and synchronous metastatic colorectal cancer. BMC Cancer. 2012;12:574.

38. Estecio MR, Gharibyan V, Shen L, Ibrahim AE, Doshi K, He R, et al. LINE-1 hypomethylation in cancer is highly variable and inversely correlated with microsatellite instability. PLoS One. 2007;2:e399.

39. Hubbard JM, Grothey A. Adolescent and young adult colorectal cancer. J Natl Compr Canc Netw. 2013;11:1219-25.

40. Mork ME, You YN, Ying J, Bannon SA, Lynch PM, Rodriguez-Bigas MA, et al. High prevalence of hereditary cancer syndromes in adolescents and young adults with colorectal cancer. J Clin Oncol. 2015:33(31):3544-9.

41. Rodriguez-Bigas MA, Mahoney MC, Weber TK, Petrelli NJ. Colorectal cancer in patients aged 30 years or younger. Surg Oncol. 1996:5:189-94.

42. Ogino S, Kawasaki T, Kirkner GJ, Loda M, Fuchs CS. CpG island methylator phenotype-low (CIMP-low) in colorectal cancer: possible associations with male sex and KRAS mutations. J Mol Diagn. 2006;8:582-8.

43. Zampieri M, Ciccarone F, Calabrese R, Franceschi C, Burkle A, Caiafa P. Reconfiguration of DNA methylation in aging. Mech Ageing Dev. 2015;151:60-70.

44. Hiltunen MO, Koistinaho J, Alhonen L, Myohanen S, Marin S, Kosma VM, et al. Hypermethylation of the WT1 and calcitonin gene promoter regions at chromosome 11p in human colorectal cancer. Br J Cancer. 1997;76:1124-30.

45. Jia Y, Guo M. Epigenetic changes in colorectal cancer. Chin J Cancer. 2013;32:21-30.

46. Ottaviano YL, Issa JP, Parl FF, Smith HS, Baylin SB, Davidson NE. Methylation of the estrogen receptor gene $\mathrm{CpG}$ island marks loss of estrogen receptor expression in human breast cancer cells. Cancer Res. 1994;54:2552-5.

47. Xu XL, Yu J, Zhang HY, Sun MH, Gu J, Du X, et al. Methylation profile of the promoter $\mathrm{CpG}$ islands of 31 genes that may contribute to colorectal carcinogenesis. World J Gastroenterol. 2004;10:3441-54.

48. Valo S, Kaur S, Ristimaki A, Renkonen-Sinisalo L, Jarvinen $H$, Mecklin JP, et al. DNA hypermethylation appears early and shows increased frequency with dysplasia in Lynch syndrome-associated colorectal adenomas and carcinomas. Clin Epigenetics. 2015;7:71. 
49. Mima K, Nishihara R, Qian ZR, Cao Y, Sukawa Y, Nowak JA, et. al., Fusobacterium nucleatum in colorectal carcinoma tissue and patient prognosis. Gut 2015. doi: 10.1136/gutjnl-2015-310101

50. Tahara T, Yamamoto E, Suzuki H, Maruyama R, Chung W, Garriga J, et al. Fusobacterium in colonic flora and molecular features of colorectal carcinoma. Cancer Res. 2014;74:1311-8.

51. Ogino S, Lochhead P, Chan AT, Nishihara R, Cho E, Wolpin BM, et al. Molecular pathological epidemiology of epigenetics: emerging integrative science to analyze environment, host, and disease. Mod Pathol. 2013;26:465-84.

52. Parsons MT, Buchanan DD, Thompson B, Young JP, Spurdle AB. Correlation of tumour BRAF mutations and MLH1 methylation with germline mismatch repair (MMR) gene mutation status: a literature review assessing utility of tumour features for MMR variant classification. J Med Genet. 2012;49:151-7.

53. Roberts SA, Gordenin DA. Hypermutation in human cancer genomes: footprints and mechanisms. Nat Rev Cancer. 2014:14:786-800.

54. Greenman C, Stephens P, Smith R, Dalgliesh GL, Hunter C, Bignell G, et al. Patterns of somatic mutation in human cancer genomes. Nature. 2007:446:153-8.

55. Hodgkinson A, Eyre-Walker A. Variation in the mutation rate across mammalian genomes. Nat Rev Genet. 2011;12:756-66.

56. Vogelstein B, Papadopoulos N, Velculescu VE, Zhou S, Diaz Jr LA, Kinzler KW. Cancer genome landscapes. Science. 2013;339:1546-58.

57. Hamilton SR, Vogelstein B, Kudo S, Riboli E, Nakamura S, Hainaut P, et al. Tumours of the Colon and Rectum. In: Hamilton SR, Aaltonen LA, editors. WHO Classification Pathology \& Genetics, Tumours of the Digestive System. Lyon: IARC Press; 2000. p. 103-44.

58. Edge SB BD, Compton CC, Fritz AG, Greene FL, Trotti A. Colon and rectum. In: Edge SB, editor. TNM classification of malignant tumors. New York: Springer; 2010. p. 173-206

59. Yang AS, Estecio MR, Doshi K, Kondo Y, Tajara EH, Issa JP. A simple method for estimating global DNA methylation using bisulfite PCR of repetitive DNA elements. Nucleic Acids Res. 2004;32:e38.

60. Stefanoli M, La Rosa S, Sahnane N, Romualdi C, Pastorino R, Marando A, et al. Prognostic relevance of aberrant DNA methylation in $\mathrm{g} 1$ and g2 pancreatic neuroendocrine tumors. Neuroendocrinol. 2014;100:26-34.

61. Furlan D, Sahnane N, Mazzoni M, Pastorino R, Carnevali I, Stefanoli M, et al. Diagnostic utility of MS-MLPA in DNA methylation profiling of adenocarcinomas and neuroendocrine carcinomas of the colon-rectum. Virchows Arch. 2013:462:47-56.

62. Sahnane N, Furlan D, Monti M, Romualdi C, Vanoli A, Vicari E, et al. Microsatellite unstable gastrointestinal neuroendocrine carcinomas: a new clinicopathologic entity. Endocr Relat Cancer. 2014;22:35-45.

63. Furlan D, Sahnane N, Bernasconi B, Frattini M, Tibiletti MG, Molinari F, et al. APC alterations are frequently involved in the pathogenesis of acinar cell carcinoma of the pancreas, mainly through gene loss and promoter hypermethylation. Virchows Arch. 2014;464:553-64.

64. Schwarz G. Estimating the dimension of a model. Ann Stat. 1978;6:461-4.

65. Raftery CFaAE. Model-based clustering, discriminant analysis, and density estimation. J Am Stat Assoc. 2002;97:611-31.

\section{Submit your next manuscript to BioMed Central and we will help you at every step:}

- We accept pre-submission inquiries

- Our selector tool helps you to find the most relevant journal

- We provide round the clock customer support

- Convenient online submission

- Thorough peer review

- Inclusion in PubMed and all major indexing services

- Maximum visibility for your research

Submit your manuscript at www.biomedcentral.com/submit 